\title{
以地理来源分组和利用表型数据构建中国小豆核心种质
}

徐宁 程须珍* 王素华王丽侠赵丹

(中国农业科学院作物科学研究所, 北京 100081)

摘 要: 中国国家种质库里保存着 4877 份小豆(Vigna angularis)种质资源,但仅有部分资源在小豆的改良中被利用。 构建小豆核心种质，不但能简化管理，而且可以提高遗传资源在小亚改良中的利用效率。根据我国小豆的地理来源进 行分组, 在分组的基础上利用表型数据, 以类平均法聚类构建了中国现有小豆核心种质, 共 435 份资源, 占资源总量 的 $8.92 \%$, 涵盖 $98.3 \%$ 的表型变异类型, 控制不同性状表型相关的相互适应的遗传复杂性在核心种质中也得到了适当 的保持。不同性状的均值 $t$ 测验、方差 $F$ 测验、表型频率分布、 $\chi^{2}$ 测验等分析表明, 所构建的小豆核心种质可以作 为整体资源的代表性样本, 在小豆种质资源的充分利用中将发挥重要作用。

关键词：小豆(Vigna angularis)；核心种质；表现型；遗传资源

\section{Establishment of an Adzuki Bean (Vigna angularis) Core Collection Based on Geographical Distribution and Phenotypic Data in China}

\author{
XU Ning, CHENG Xu-Zhen*, WANG Su-Hua, WANG Li-Xia, and ZHAO Dan \\ (Institute of Crop Sciences, Chinese Academy of Agricultural Sciences, Beijing 100081, China)
}

\begin{abstract}
A total of 4877 accessions of adzuki bean (Vigna angularis) are conserved in National Germplasm Conservation Center in China, but only a few of which have been used in adzuki bean improvement programme. Establishing a core collection of adzuki bean can simplify management and enhance utilization of genetic resources in adzuki bean research programmes. In this paper, Unweighted Pair-Group Method Using Arithmetic Averages (UPGMA) was used to establish an adzuki bean core collection based on geographical distribution and phenotypic data. Finally, $8.92 \%$ of adzuki bean accessions were selected from entire collection as a core collection of 435 accessions sharing $98.3 \%$ phenotypes with the entire collection. The phenotypic correlations among different traits that may be under the control of co-adapted gene complexes were also preserved in the core collection. By different statistical methods such as comparison of mean using $t$-test, variance using $F$-test, phenotypic frequency distributions using $\chi^{2}$-test for the different traits, the core collection was validated to be a representative sample of the entire collection. This core collection will play an important role in the utilization of adzuki bean genetic resources.
\end{abstract}

Keywords: Adzuki bean (Vigna angularis); Core collection; Phenotypes; Genetic resources

小豆[Vigna angularis (Willd) Ohwi \& Ohashi] 是高蛋白、低脂肪、医食同源作物，其籽粒含有人 体所必需的铁、钙、磷等微量元素和 18 种氨基酸, 其 中赖氨酸含量高达 $1.8 \%{ }^{[1]}$, 还有清热解毒、保肝明 目、降低血压、防止动脉硬化等多种医疗功效 ${ }^{[2]}$ 。 在我国小豆是六大食用豆类之一 ${ }^{[3]}$, 有着 2000 多年 的栽培历史 ${ }^{[2]}$, 大部分省区都种植，以华北、黄河中 下游、东北地区种植面积最大 ${ }^{[4]}$ 。全世界共有 20 多
个国家种植小互, 中国种植面积最大 ${ }^{[5]}$, 日本和韩国 次之, 美国、印度、新西兰等国也有一定种植面积。

作物种质资源的收集是保护、评价和利用种质 资源的基础, 在过去二、三十年里, 世界各国都非常 重视这一工作, 大量资源被集中在了种质库里 ${ }^{[6]}$ 。我 国小豆种质资源已超过 4800 多份, 占世界第一位, 但由于缺乏适当的评价数据而阻碍了对小亘资源的 利用。这一现状在小麦 ${ }^{[7]}$ 、春大麦 ${ }^{[8]} 、$ 玉米 $^{[9]}$ 、花生 ${ }^{[10]}$ 、

基金项目: 作物种质资源保护项目(NB04-07); 国家科技基础条件平台建设项目(2004-06DKA); “ 十一五” 国家科技支撑计划(2006BAD02B08); 农业科研专项资金 (nykyzx07-017)

作者简介: 徐宁(1981-), 男, 硕士研究生, 主要从事小亘遗传多样性研究。E-mail: xunig2008@163.com

“通讯作者(Corresponding author): 程须珍, 研究员, 主要从事食用豆品种资源研究。Tel:010-62189159; E-mail: Chengxz@caas.net.cn Received(收稿日期): 2007-12-04; Accepted(接受日期): 2008-03-12. 
木豆 ${ }^{[11]}$ 、鹰嘴豆 ${ }^{[12]}$ 和珍珠粟 ${ }^{[13]}$ 等种质资源中都曾有 报道。在育种研究中, 数量性状在很大程度上受环 境的影响, 需要多年多点的重复试验对其进行评价, 因此很有必要优化庞大的资源群体，使其达到能够 很好管理的规模, 这样通过试验才能得出准确的数 据, 加强对种质资源的利用。鉴于以上认识, 1984 年 Frankel ${ }^{[14]}$ 提出了构建核心种质的方法, 旨在用 最少的种质资源样品最大程度地代表种质资源的 遗传多样性。此后, 已在多种作物上构建了核心种 质，包括花生 ${ }^{[15]}$ 、高粱 ${ }^{[16]}$ 、鹰嘴豆 ${ }^{[17]}$ 、小麦 ${ }^{[18]}$ 、 玉米 $^{[19]}$ 、木豆 ${ }^{[20]}$ 、珍珠粟 ${ }^{[13]}$ 、苜宿 ${ }^{[21]}$ 等。我国国 家重点基础研究发展规划项目 “农作物核心种质构 建、重要新基因发掘与有效利用研究”提出了核心 种质构建一重要新基因发掘一一基因克隆的技

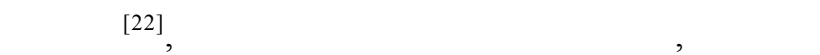
核心种质的构建不仅是此项目的基础, 而且是我国 种质资源深入研究及有效开发利用的基础, 对作物 育种以至农业研究的许多学科, 具有深远的科学与 经济意义。此项目确定我国具有较好研究基础的小 麦、水稻、大豆三大作物为重点研究对象, 并且建 立了各自的核心种质 ${ }^{[18,23-24]}$ 。利用核心种质能够对 种质资源进行更为广泛的评价, 特别是对所需性状 的研究更加经济有效, 因此核心种质在作物改良中 有着极其重要的价值。

Frankel 和 Brown ${ }^{[25]}$ 、Brown ${ }^{[26]}$ 提出可以利用地 理来源和农艺性状数据构建核心种质。本研究将利 用地理来源和表现型数据从保存在中国国家种质库
的 4877 份小亘资源中选出核心种质。虽然, 王述民 ${ }^{[27]}$ 在 2001 年已经初步建立了小亘核心种质, 但当时小 豆种质资源仅为 3946 份, 对所构建核心种质的代 表性检验也比较单一, 并且没有把广西、广东、台 湾和海南等资源份数极少的省份包括在内, 而笔者 发现, 广西资源在表型上有自己独特的变异类型, 因此有必要把资源份数极少的省份也考虑在内, 同 时本文亦把日本、澳大利亚、朝鲜共计 91 份资源包 括在内, 这些资源同样能在生产中发挥作用。加之 核心种质本身也有动态性特点 ${ }^{[28]}$, 重新建立一套适 应当前生产需要的小五核心种质势在必行。

\section{1 材料与方法}

\section{1 试验材料}

小豆核心种质的建立主要依据中国国家种质库 4877 份小互种质资源的鉴定数据, 取自国家种质资 源数据库。农艺性状包括生长习性、结荚习性、荚 色、粒色、粒形、株高、生育日数、百粒重、分枝 数、单株荚数、单荚粒数、荚长等 12 项, 营养品质 性状包括粗蛋白、粗脂肪、总淀粉等 3 项。

\section{2 数据标准化}

对小亚非数值性状如粒色、生长习性、粒形等 予以赋值 (代码值), 如粒色, 1=白, $2=$ 黄, $3=$ 红, 4=绿, $5=$ 黑, $6=$ 褐, $7=$ 花纹, $8=$ 花斑(表 1)。对数值性状如株 高、百粒重等进行 10 级分类, 1 级 $\leq X-2 \delta, 10$ 级 $>$ $X+2 \delta$, 中间每级间差 $0.5 \delta, X$ 为各性状平均值, $\delta$ 为标 准差。

表 1 小豆非数值型性状赋值

Table 1 Coden designed for qualitative traits in adzuki bean

\begin{tabular}{|c|c|}
\hline 性状 Trait & 赋值 Coden of qualitative traits \\
\hline 生长习性 Growth habit & 直立 $=1$, 半蔓生 $=2$, 蔓生 $=3 \quad$ Erect $=1$, Semi-erect $=2$, Prostrate $=3$ \\
\hline 结荚习性 Habit of pod-setting & Termination $=1$, Semi-termination $=2$, Intermination $=3$ \\
\hline 荚色 Pod color & 黄白 $=1$, 棕 $=2$, 黑 $=3$ Yellowish white $=1$, Brown=2, Black $=3$ \\
\hline 粒色 Seed coat color & $\begin{array}{l}\text { 白 }=1, \text { 黄 }=2, \text { 红 }=3, \text { 绿 }=4, \text { 黑 }=5, \text { 褐 }=6, \text { 花纹 }=7, \text { 花斑 }=8 \\
\text { White }=1, \text { Yellow }=2, \text { Red }=3 \text {, Green }=4, \text { Black }=5, \text { Brown }=6 \text {, Pattern }=7 \text {, Piebald }=8\end{array}$ \\
\hline 粒形 Seed shape & 长圆柱 $=1$, 短圆柱 $=2$, 球形 $=3$ Long-cylinder $=1$, Short-cylinder $=2$, Spherical $=3$ \\
\hline
\end{tabular}

\section{3 核心种质的构建}

以省 (直辖市、自治区)为单位分组 (日本、澳大 利亚、朝鲜资源份数少，将其看作 3 个不同的组)，在 分组的基础上, 利用 12 个农艺性状和 3 个营养品质 性状数据进行聚类, 种质间距离为欧式距离, 用类 平均法(UPGMA)和 NTSYSpc-2.10t 软件进行聚类, 用 999 代替缺失数据。对每个类群利用比例法取样
并做适当调整(表 2), 比例法是指组内取样比例与组 内材料量占整个资源材料量的比例相一致。在构建 鹰嘴豆 ${ }^{[17]}$ 、中国栽培大豆 ${ }^{[24]}$ 、印度绿豆、扁豆 ${ }^{[29]}$ 等豆类核心种质时都采用了比例法。

\section{4 核心种质代表性检测}

通过对核心种质与全部种质间相关参数的比较, 判断所构建核心种质是否有代表性。以 $t$ 测验检验 
表 2 各组资源份数占全部资源的比例以及各组取样份数

Table 2 Proportion of accessions from 27 groups in the entire collection and the number of core sample in each group

\begin{tabular}{|c|c|c|c|c|c|c|}
\hline \multirow{2}{*}{$\begin{array}{l}\text { 组别 } \\
\text { Group }\end{array}$} & \multicolumn{3}{|c|}{ 全部种质 Entire collection } & \multicolumn{3}{|c|}{ 核心种质 Core collection } \\
\hline & 数目 & Number & 比例 Rate $(\%)$ & 数目 & Number & 比例 Rate $(\%)$ \\
\hline 中国山西 Shanxi, China & & 603 & 12.36 & & 75 & 17.24 \\
\hline 中国河北 Hebei, China & & 437 & 8.96 & & 40 & 9.20 \\
\hline 中国湖北 Hubei, China & & 418 & 8.57 & & 40 & 9.20 \\
\hline 中国陕西 Shaanxi, China & & 383 & 7.85 & & 30 & 6.90 \\
\hline 中国北京 Beijing, China & & 381 & 7.81 & & 30 & 6.90 \\
\hline 中国河南 Henan, China & & 371 & 7.61 & & 34 & 7.82 \\
\hline 中国安徽 Anhui, China & & 335 & 6.87 & & 25 & 5.75 \\
\hline 中国吉林 Jilin, China & & 289 & 5.93 & & 22 & 5.06 \\
\hline 中国黑龙江 Heilongjiang, China & & 262 & 5.37 & & 16 & 3.68 \\
\hline 中国山东 Shandong, China & & 229 & 4.70 & & 18 & 4.14 \\
\hline 中国四川 Sichuan, China & & 186 & 3.81 & & 8 & 1.84 \\
\hline 中国辽宁 Liaoning, China & & 175 & 3.59 & & 12 & 2.76 \\
\hline 中国贵州 Guizhou, China & & 150 & 3.08 & & 9 & 2.07 \\
\hline 中国内蒙古 Inner Mongolia, China & & 145 & 2.97 & & 10 & 2.30 \\
\hline 中国江苏 Jiangsu, China & & 141 & 2.89 & & 11 & 2.53 \\
\hline 中国云南 Yunnan, China & & 67 & 1.37 & & 6 & 1.38 \\
\hline 中国天津 Tianjin, China & & 64 & 1.31 & & 8 & 1.84 \\
\hline 中国甘肃 Gansu, China & & 62 & 1.27 & & 6 & 1.38 \\
\hline 日本 Japan & & 60 & 1.23 & & 7 & 1.61 \\
\hline 中国湖南 Hunan, China & & 56 & 1.15 & & 8 & 1.84 \\
\hline 澳大利亚 Australia & & 30 & 0.62 & & 5 & 1.15 \\
\hline 中国宁夏 Ningxia, China & & 17 & 0.35 & & 5 & 1.15 \\
\hline 中国广西 Guangxi, China & & 8 & 0.16 & & 4 & 0.92 \\
\hline 中国广东 Guangdong, China & & 3 & 0.06 & & 2 & 0.46 \\
\hline 中国台湾 Taiwan, China & & 3 & 0.06 & & 2 & 0.46 \\
\hline 中国海南 Hainan, China & & 1 & 0.02 & & 1 & 0.23 \\
\hline 朝鲜 Korea & & 1 & 0.02 & & 1 & 0.23 \\
\hline 总计 Total & & & & & & \\
\hline
\end{tabular}

两者数量性状均值是否有差异, $F$ 测验检测两者数 量性状变异是否同质, $\chi^{2}$ 测验检测两者的表型分布 是否一致, 表型相关分析判断各性状复杂的相关性 在核心种质中是否得到了相应保持, 利用 Shannon-Weaver 遗传多样性指数 $(H)$ 评价表现型遗 传多样性 ${ }^{[30]}$ 。

$$
H^{\prime}=-\sum_{i=1}^{s} P_{i} \ln P_{i}
$$

其中, $p_{i}$ 为某一性状第 $i$ 级别内材料份数占总份数的 百分比。

表型保留比例(ratio of phenotype retained, RPR) 用于检测核心种质中是否保留了足够的变异, 是检 验核心种质实用性的有效参数 ${ }^{[31]}$ 。

$$
R P R=\frac{\sum_{i}^{s} M_{i}}{\sum_{i}^{s} M_{i o}}
$$

其中, $M_{i o}$ 为原始库中第 $i$ 个性状的表现型个数, $M_{i}$ 为所得核心种质中第 $i$ 个性状的表现型个数。以 SAS 统计分析软件进行 $t$ 测验、 $F$ 测验和相关分析, 其余 计算均在 Microsoft Excel 中完成。

\section{2 结果与分析}

\section{$2.1 t$ 验测、 $F$ 测验以及极差分析}

在 10 个数量性状中, $t$ 测验检测到单株荚数、单 荚粒数两个性状的平均值在核心种质与全部种质中 
存在着显著差异(表 3)。经 $F$ 测验， 8 个数量性状的 方差为齐性, 其余株高、荚长两个性状的方差在核 心种质中高于全部种质, 表明核心种质获得了更大 的变异。大部分性状的极值材料都被保存在了核心 种质中(表 3), 生育日数、株高、单株荚数、荚长、 百粒重、粗蛋白、粗脂肪 7 个性状的极差符合度在 $85 \%$ 和 $100 \%$ 之间，分枝数、单荚粒数、总淀粉 3 个 性状的极差符合度在 $71 \%$ 和 $83 \%$ 之间。因此, 所选 核心种质基本能够代表全部种质。

\section{$2.2 \chi^{2}$ 测验}

对全部种质与核心种质 15 个性状的频率分布 进行 $\chi^{2}$ 测验, 除株高、百粒重两个性状差异显著外, 其余 13 个性状差异都不显著(表 4)。对频率分布分析 发现, 株高第 2 级、第 5 级、第 9 级材料占全部种
质的 $4.65 \% 、 16.99 \% 、 4.53 \%$ ，而分别占核心种质的 $2.78 \% 、 20.88 \% 、 1.62 \%$ (表 5)。百粒重第 2 级材料占 全部种质的 $4.05 \%$, 而占核心种质的 $1.39 \%$ (表 5)。 因此, 在完善核心种质时, 对于株高来说应该适当 增加比较极端的材料, 而减少中间材料; 对于百粒 重来说应该适当增加较轻的材料。5 个质量性状的 频率分布比较一致(表 6)。

\section{3 遗传多样性指数分析}

15 个性状的遗传多样性指数在全部种质与核心 种质中相似(表 7), 核心种质平均遗传多样性指数为 $1.604 \pm 0.141$, 全部种质平均遗传多样性指数为 $1.616 \pm 0.148$, 表明所选核心种质具有较好的代表 性。另外, 结荚习性的遗传多样性指数在全部种质 (0.498)与核心种质 $(0.531)$ 中相对来说都很低。

表 3 小豆全部种质与核心种质 10 个数量性状平均值、极差、方差的比较

Table 3 Comparison of mean, range, and variance for ten quantitative traits in the entire adzuki bean collection and its core collection

\begin{tabular}{|c|c|c|c|c|c|c|c|c|}
\hline \multirow{2}{*}{$\begin{array}{l}\text { 性状 } \\
\text { Trait }\end{array}$} & \multicolumn{3}{|c|}{ 全部种质 Entire collection } & \multicolumn{5}{|c|}{ 核心种质 Core collection } \\
\hline & $\begin{array}{l}\text { 平均值 } \\
\text { Mean }\end{array}$ & $\begin{array}{c}\text { 极差 } \\
\text { Range }\end{array}$ & $\begin{array}{c}\text { 方差 } \\
\text { Variance }\end{array}$ & $\begin{array}{l}\text { 平均值 } \\
\text { Mean }\end{array}$ & $\begin{array}{r}t \text { 测验 } \\
t \text {-test }\end{array}$ & $\begin{array}{c}\text { 极差 } \\
\text { Range }\end{array}$ & $\begin{array}{c}\text { 方差 } \\
\text { Variance }\end{array}$ & $\begin{array}{l}F \text { 测验 } \\
F \text {-test }\end{array}$ \\
\hline 生育日数 Growth period(d) & $119.58 \pm 0.31$ & $71-196$ & 451.40 & $119.28 \pm 1.02$ & NS & $72-196$ & 442.70 & NS \\
\hline 株高 Plant height(cm) & $76.39 \pm 0.41$ & $9-366$ & 912.60 & $78.60 \pm 1.59$ & NS & $9-366$ & 1095.50 & * \\
\hline 分枝数 Number of branches & $4.33 \pm 0.04$ & $0-32.30$ & 6.03 & $4.52 \pm 0.13$ & NS & $0.20-25.40$ & 6.63 & NS \\
\hline 单株荚数 Number of pods per plant & $30.61 \pm 0.26$ & $1.30-150.20$ & 3.05 & $33.04 \pm 1.02$ & $*$ & $2.00-150.20$ & 3.16 & NS \\
\hline 单荚粒数 Number of seeds per pod & $7.42 \pm 0.03$ & $0.50-23.80$ & 3.05 & $7.59 \pm 0.09$ & $*$ & $2.40-19.10$ & 3.16 & NS \\
\hline 荚长 Pod length(cm) & $7.86 \pm 0.02$ & $3.80-17.80$ & 1.17 & $7.98 \pm 0.06$ & NS & $3.80-16.10$ & 1.38 & $*$ \\
\hline 百粒重 100-seed weight(g) & $9.59 \pm 0.04$ & $1.80-20.00$ & 6.99 & $9.35 \pm 0.13$ & NS & $1.80-18.90$ & 6.98 & NS \\
\hline 粗蛋白 Crude protein content $(\%)$ & $22.72 \pm 0.04$ & $16.30-29.20$ & 3.29 & $22.77 \pm 0.13$ & NS & $16.33-28.35$ & 3.56 & NS \\
\hline 粗脂肪 Crude fat content(\%) & $0.59 \pm 0.01$ & $0.04-2.65$ & 0.12 & $0.59 \pm 0.03$ & NS & $0.10-2.48$ & 0.14 & NS \\
\hline 总淀粉 Crude starch content(\%) & $53.14 \pm 0.04$ & $39.38-59.89$ & 4.80 & $53.21 \pm 0.15$ & NS & $41.83-58.83$ & 4.86 & NS \\
\hline
\end{tabular}

*: 0.05 水平上差异显著; NS: 0.05 水平上差异不显著。

*: significantly different at 0.05 probability level; NS: not significant at 0.05 probability level.

表 4 核心种质与全部种质 15 个性状频率分布 $\chi^{2}$ 测验

Table 4 Frequency distribution of 15 traits between core collection and entire collection by $\chi^{2}$ test

\begin{tabular}{|c|c|c|c|c|c|c|c|}
\hline \multirow[b]{2}{*}{$\begin{array}{l}\text { 性状 } \\
\text { Trait }\end{array}$} & \multicolumn{3}{|c|}{ 分布频率 Frequency of distribution } & \multirow[b]{2}{*}{$\begin{array}{l}\text { 性状 } \\
\text { Trait }\end{array}$} & \multicolumn{3}{|c|}{ 分布频率 Frequency of distribution } \\
\hline & $\begin{array}{c}\text { 级数 } \\
\text { Number } \\
\text { of classes }\end{array}$ & $\chi^{2}$ & $P$ & & $\begin{array}{c}\text { 级数 } \\
\text { Number of } \\
\text { classes }\end{array}$ & $\chi^{2}$ & $P$ \\
\hline 生育日数 Growth period(d) & 10 & 5.19 & $0.80-0.90$ & 粗脂肪 Crude fat content $(\%)$ & 10 & 3.67 & $0.90-0.95$ \\
\hline 株高 Plant height(cm) & 10 & 18.17 & $0.01-0.05^{*}$ & 总淀粉 Crude starch content $(\%)$ & 10 & 5.61 & $0.70-0.80$ \\
\hline 分枝数 Number of branches & 10 & 3.07 & $0.95-0.99$ & 生长习性 Growth habit & 3 & 0.83 & $0.50-0.70$ \\
\hline 单株荚数 Number of pods per plant & 10 & 7.64 & $0.50-0.70$ & 结荚习性 Habit of pod-setting & 3 & 1.91 & $0.30-0.50$ \\
\hline 单荚粒数 Number of seeds per pod & 10 & 10.63 & $0.30-0.50$ & 荚色 Pod color & 3 & 4.51 & $0.10-0.20$ \\
\hline 荚长 Pod length(cm) & 10 & 13.73 & $0.10-0.20$ & 粒色 Seed color & 8 & 8.82 & $0.20-0.30$ \\
\hline 百粒重 100-seed weight(g) & 10 & 17.29 & $0.01-0.05^{*}$ & 粒形 Seed shape & 3 & 5.27 & $0.05-0.10$ \\
\hline 粗蛋白 Crude protein content $(\%)$ & 10 & 8.21 & $0.50-0.70$ & & & & \\
\hline
\end{tabular}

${ }^{*}: 0.05$ 水平上差异显著。": significantly different at 0.05 probability level. 
表 5 核心种质与全部种质数量性状各等级频率分布

Table 5 Frequency distribution of quantitative traits of each class both in core collection and entire collection(\%)

\begin{tabular}{|c|c|c|c|c|c|c|c|c|c|c|c|}
\hline \multirow{2}{*}{$\begin{array}{l}\text { 性状 } \\
\text { Trait }\end{array}$} & & \multicolumn{10}{|c|}{ 级别 Class } \\
\hline & & 1 & 2 & 3 & 4 & 5 & 6 & 7 & 8 & 9 & 10 \\
\hline \multirow[t]{2}{*}{ 生育日数 Growth period } & 全部 Entire & 0.17 & 1.96 & 10.41 & 21.33 & 27.02 & 12.14 & 9.02 & 10.33 & 2.70 & 4.91 \\
\hline & 核心 Core & 0.23 & 3.03 & 9.79 & 19.58 & 27.27 & 11.89 & 10.49 & 10.02 & 3.26 & 4.43 \\
\hline \multirow[t]{2}{*}{ 株高 Plant height } & 全部 Entire & 0.21 & 4.65 & 12.17 & 17.76 & 16.99 & 18.10 & 14.19 & 8.86 & 4.53 & 2.55 \\
\hline & 核心 Core & 0.23 & 2.78 & 9.98 & 19.72 & 20.88 & 19.26 & 13.92 & 8.82 & 1.62 & 2.78 \\
\hline \multirow[t]{2}{*}{ 分枝数 Number of branches } & 全部 Entire & 0 & 1.86 & 8.77 & 19.71 & 25.37 & 19.28 & 14.24 & 7.79 & 1.75 & 1.23 \\
\hline & 核心 Core & 0 & 1.73 & 8.91 & 19.06 & 26.49 & 20.05 & 13.12 & 6.93 & 1.73 & 1.98 \\
\hline \multirow[t]{2}{*}{ 单株荚数 Number of pods per plant } & 全部 Entire & 0 & 0.21 & 11.66 & 27.52 & 20.23 & 13.71 & 10.21 & 7.62 & 4.57 & 4.27 \\
\hline & 核心 Core & 0 & 0 & 11.40 & 25.35 & 24.65 & 12.56 & 10.93 & 6.51 & 3.95 & 4.65 \\
\hline \multirow[t]{2}{*}{ 单荚粒数 Number of seeds per pod } & 全部 Entire & 1.94 & 7.30 & 6.65 & 12.01 & 19.23 & 24.03 & 16.47 & 7.57 & 2.99 & 1.81 \\
\hline & 核心 Core & 2.79 & 5.35 & 6.05 & 14.88 & 16.74 & 23.72 & 19.07 & 7.21 & 2.56 & 1.63 \\
\hline \multirow[t]{2}{*}{ 荚长 Pod length } & 全部 Entire & 1.42 & 4.76 & 8.97 & 16.60 & 18.26 & 20.27 & 15.94 & 7.35 & 3.74 & 2.68 \\
\hline & 核心 Core & 1.48 & 4.20 & 10.86 & 12.59 & 16.79 & 25.19 & 14.81 & 8.64 & 3.70 & 1.73 \\
\hline \multirow[t]{2}{*}{ 百粒重 100-seed weight } & 全部 Entire & 0.84 & 4.05 & 11.64 & 16.81 & 17.44 & 19.24 & 13.71 & 8.99 & 4.43 & 2.85 \\
\hline & 核心 Core & 1.62 & 1.39 & 12.76 & 18.10 & 19.49 & 18.33 & 11.37 & 9.51 & 3.48 & 3.94 \\
\hline \multirow[t]{2}{*}{ 粗蛋白 Crude protein content } & 全部 Entire & 1.58 & 4.73 & 9.34 & 16.01 & 19.13 & 19.57 & 15.04 & 7.97 & 3.52 & 3.11 \\
\hline & 核心 Core & 0.90 & 5.41 & 10.36 & 14.86 & 14.86 & 25.68 & 14.86 & 7.21 & 2.70 & 3.15 \\
\hline \multirow[t]{2}{*}{ 粗脂肪 Crud fat content } & 全部 Entire & 0 & 0.07 & 5.86 & 29.24 & 28.28 & 16.76 & 9.93 & 3.31 & 1.59 & 4.97 \\
\hline & 核心 Core & 0 & 0 & 3.88 & 27.13 & 34.88 & 15.50 & 10.08 & 2.33 & 1.55 & 4.65 \\
\hline \multirow[t]{2}{*}{ 总淀粉 Crude starch content } & 全部 Entire & 2.90 & 4.73 & 9.26 & 11.54 & 17.41 & 22.63 & 16.80 & 9.50 & 3.92 & 1.31 \\
\hline & 核心 Core & 3.18 & 2.73 & 10.00 & 9.55 & 19.09 & 25.45 & 17.27 & 7.27 & 3.64 & 1.82 \\
\hline
\end{tabular}

表 6 核心种质与全部种质质量性状各等级频率分布

Table 6 Frequency distribution of qualitative traits of each class both in core collection and entire collection(\%)

\begin{tabular}{|c|c|c|c|c|c|c|c|c|c|}
\hline \multirow{2}{*}{$\begin{array}{l}\text { 性状 } \\
\text { Trait }\end{array}$} & & \multicolumn{8}{|c|}{ 级别 Class } \\
\hline & & 1 & 2 & 3 & 4 & 5 & 6 & 7 & 8 \\
\hline \multirow[t]{2}{*}{ 生长习性 Growth habit } & 全部 Entire & 38.94 & 45.73 & 15.33 & & & & & \\
\hline & 核心 Core & 37.79 & 45.24 & 16.97 & & & & & \\
\hline \multirow{2}{*}{ 结荚习性 Habit of pod-setting } & 全部 Entire & 16.13 & 1.04 & 82.83 & & & & & \\
\hline & 核心 Core & 16.46 & 1.72 & 81.82 & & & & & \\
\hline \multirow[t]{2}{*}{ 荚色 Pod color } & 全部 Entire & 72.33 & 22.49 & 5.19 & & & & & \\
\hline & 核心 Core & 71.84 & 20.76 & 7.40 & & & & & \\
\hline \multirow[t]{2}{*}{ 粒色 Seed color } & 全部 Entire & 24.05 & 4.85 & 52.65 & 8.58 & 0.50 & 0.33 & 6.81 & 2.23 \\
\hline & 核心 Core & 25.17 & 3.46 & 50.12 & 9.01 & 0.92 & 0.46 & 9.24 & 1.62 \\
\hline \multirow[t]{2}{*}{ 粒形 Seed shape } & 全部 Entire & 18.78 & 69.84 & 11.38 & & & & & \\
\hline & 核心 Core & 23.15 & 66.75 & 10.10 & & & & & \\
\hline
\end{tabular}

表 7 小五全部种质与核心种质 15 个性状 Shannon-Weaver 遗传多样性指数比较

Table 7 Comparison of Shannon-Weaver diversity indices for 15 traits between the entire and core collection of adzuki bean

\begin{tabular}{|c|c|c|c|c|c|}
\hline $\begin{array}{r}\text { 性状 } \\
\text { Trait } \\
\end{array}$ & $\begin{array}{c}\text { 全部种质 } \\
\text { Entire collection }\end{array}$ & $\begin{array}{c}\text { 核心种质 } \\
\text { Core collection }\end{array}$ & $\begin{array}{l}\text { 性状 } \\
\text { Trait }\end{array}$ & $\begin{array}{c}\text { 全部种质 } \\
\text { Entire collection }\end{array}$ & $\begin{array}{c}\text { 核心种质 } \\
\text { Core collection }\end{array}$ \\
\hline 生育日数 Growth period(d) & 1.960 & 1.991 & 粗脂肪 Crude fat content $(\%)$ & 1.744 & 1.662 \\
\hline 株高 Plant height(cm) & 2.055 & 1.960 & 总淀粉 Crude starch content(\%) & 2.064 & 2.014 \\
\hline 分枝数 Number of branches & 1.874 & 1.875 & 生长习性 Growth habit & 1.013 & 1.028 \\
\hline 单株荚数 Number of pods per plant & 1.919 & 1.891 & 结荚习性 Habit of pod-setting & 0.498 & 0.531 \\
\hline 单荚粒数 Number of seeds per pod & 2.032 & 2.017 & 荚色 Pod color & 0.723 & 0.757 \\
\hline 荚长 Pod length $(\mathrm{cm})$ & 2.058 & 2.031 & 粒色 Seed coat color & 1.351 & 1.382 \\
\hline 百粒重 100-seed weight(g) & 2.070 & 2.044 & 粒形 Seed shape & 0.812 & 0.840 \\
\hline 粗蛋白 Crude protein content(\%) & 2.072 & 2.030 & 平均值 \pm 标准误 $\operatorname{Mean} \pm S E$ & $1.616 \pm 0.148$ & $1.604 \pm 0.141$ \\
\hline
\end{tabular}




\section{4 表型相关分析}

在构建核心种质时，合适的取样策略应考虑相 互适应的复杂表型相关性的保持情况 ${ }^{[32]}$ 。在小豆全 部种质中, 粗脂肪与生育日数、株高、分枝数、单 株荚数、荚长、百粒重、总淀粉的相关性存在显著 差异, 而在核心种质中, 粗脂肪与生育日数、总淀粉
的相关性存在显著差异, 与其他 5 个性状的相关性 不存在显著差异(表 8)。除了粗脂肪这一性状与其他 性状的相关性没有得到足够的保持外, 其他性状间 表型相关与全部种质性状间表型相关基本一致(表 8), 控制这些相关的相互适应的遗传复杂性得到了 适当并且充分的保持。

表 8 全部种质(上三角)与核心种质(下三角)数量性状表型相关性

Table 8 Phenotypic correlations between quantitative traits in entire collection(above diagonal) and core collection(below diagonal)

\begin{tabular}{ccccccccccc}
\hline 性状 Trait & GP & PH & NB & NPP & NSP & PL & SW & CPC & CFC & CSC \\
\hline GP & & $0.290^{*}$ & $-0.095^{*}$ & $-0.211^{*}$ & -0.015 & $0.052^{*}$ & $-0.092^{*}$ & $-0.188^{*}$ & $0.413^{*}$ & $0.088^{*}$ \\
PH & $0.254^{*}$ & & $0.216^{*}$ & $0.253^{*}$ & $0.195^{*}$ & $0.264^{*}$ & -0.001 & 0.038 & $-0.096^{*}$ & 0.019 \\
NB & $-0.107^{*}$ & $0.219^{*}$ & & $0.449^{*}$ & $0.306^{*}$ & $0.094^{*}$ & 0.006 & $0.282^{*}$ & $-0.077^{*}$ & $-0.267^{*}$ \\
NPP & $-0.116^{*}$ & $0.229^{*}$ & $0.396^{*}$ & & $0.282^{*}$ & $0.182^{*}$ & -0.013 & $0.155^{*}$ & $-0.153^{*}$ & $-0.107^{*}$ \\
NSP & 0.014 & $0.225^{*}$ & $0.268^{*}$ & $0.326^{*}$ & & $0.365^{*}$ & $-0.169^{*}$ & $0.153^{*}$ & -0.006 & $-0.092^{*}$ \\
PL & $0.114^{*}$ & $0.256^{*}$ & 0.079 & $0.179^{*}$ & $0.409^{*}$ & & $0.205^{*}$ & 0.036 & $-0.127^{*}$ & $0.098^{*}$ \\
SW & $-0.188^{*}$ & -0.076 & 0.046 & -0.004 & -0.081 & $0.216^{*}$ & & $0.157^{*}$ & $-0.056^{*}$ & 0.015 \\
CPC & $-0.235^{*}$ & -0.000 & $0.308^{*}$ & $0.225^{*}$ & $0.138^{*}$ & 0.029 & 0.131 & & 0.051 & $-0.687^{*}$ \\
CFC & $0.461^{*}$ & 0.058 & -0.119 & -0.155 & -0.112 & -0.168 & -0.119 & -0.026 & -0.19 & $-0.359^{*}$ \\
CSC & $0.158^{*}$ & 0.028 & $-0.261^{*}$ & -0.558 & -0.016 & $0.158^{*}$ & 0.056 & $-0.659^{*}$ & $-0.200^{*}$ &
\end{tabular}

": 表型相关在 0.05 达到显著水平。 $\mathrm{GP}$ : 生育日数; PH：株高; NB：分枝数; NPP：单株荚数; NSP：单荚粒数; PL：荚长; SW：百 粒重; CPC: 粗蛋白; CFC: 粗脂肪; CSC: 总淀粉。

*: significance of phenotypic correlations at the 0.05 probability level. GP: growth period; PH: plant height; NB: number of branches; NPP: number of pods per plant; NSP: number of seeds per pod; PL: pod length; SW: 100-seed weight; CPC: crude protein content; CFC: crude fat content; CSC: crude starch content.

\section{5 表型保留比例分析}

表型保留比例表示核心种质中所保留表型值的 数量占原始库中表型总量的比例 ${ }^{[31]}$, 由表 5 和表 6 计算出的小豆核心种质的表型保留比例为 $98.3 \%$, 表明此核心种质保留了全部种质足够丰富的变异, 有利于小亘的育种工作。

\section{3 讨论}

王述民 ${ }^{[27]}$ 在构建小亘核心种质时确定每组的 取样比例均为 $10 \%$, 共选出 408 份入选核心种质, 占总体资源的 $10.3 \%$, 而本研究采用比例法从每组 资源占总体资源的比例这一角度确定各组的取样 比例, 共选出 435 份入选核心种质, 占总体资源的 $8.92 \%$ 。代表性检验方面，王述民只用了平均值、 极值、标准差、变异系数和遗传多样性指数这 5 个 参数, 而本研究除了这些参数外, 对全部种质与核 心种质 15 个性状的频率分布进行 $\chi^{2}$ 测验、表型相 关分析和表型保留比例分析, 从遗传变异和遗传结 构上评价了所得核心种质的代表性和实用性, 这无 疑更有说服力。
构建核心种质的目的是为更好地对遗传资源进 行保存和利用 ${ }^{[6]}$ 。本研究所构建的核心种质是对小 豆资源做进一步研究和开发利用的重要切入点。例 如, 可以利用同工酶或 DNA 分子标记对小豆核心种 质进行遗传多样性分析, 根据评价结果指导下一步 种质资源的考察方向以及评价利用的重点(假如根 据分子数据发现某省份的遗传变异十分丰富, 而在 全部资源中该省份的资源数目很有限, 则可加强该 省份资源的收集力度)。此外，小豆核心种质能有效 用于特定抗性基因材料的篮选，包括抗虫性、抗病 性、抗旱性、抗寒性等等。目前，已对全部种质资 源中的 915 份材料做了抗旱性鉴定 ${ }^{[33]}$, 如对剩余的 近 4000 份资源继续做抗旱性鉴定，既费时又费力。 然而，用小豆核心种质可在相对短的时期内从整体 资源的角度进行篮选，既节省时间又节省人力、物 力。利用核心种质对所需性状进行评价在豆科其他 作物中已有不少例子, 如花生后期叶斑病抗性鉴

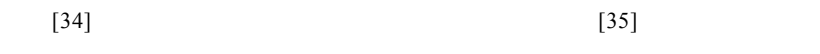
病抗性鉴定 ${ }^{[36]}$ 、花生熟性鉴定 ${ }^{[37]}$ 等等。所需性状的 材料被鉴定出后, 育种家可以对未被选入核心种质 
但与被选材料聚类在一起的资源进行评价, 也可能 篮选出具有相应抗性或者携带其他优异基因的材料, 从而拓宽育种材料的遗传基础。

我国是小亘的起源国, 资源占有量居世界第一 位, 但小亘一直被看作是“小作物”, 基础性研究相 对比较薄弱。小亚核心种质的建立应当被看作新的 起点, 对这套种质资源进行全面的鉴定、评价, 逐步 对其完善, 然后进行充分的研究和利用, 这是今后 的重点工作。在此基础上, 我们将可按“核心种质构 建 $\rightarrow$ 重要新基因发掘 $\rightarrow$ 基因克隆”的技术路线，对小 豆基因资源进行充分发掘和有效利用。

\section{4 结论}

以表现型数据构建了中国小豆核心种质，它可 代表全部资源的遗传多样性, 为小亚种质资源的进 一步研究和利用提供有效工具。

\section{References}

[1] Fu C-Z(傅翠真). Determination and Evaluation on Nutrition Quality for Chinese Food Legumes(中国食用豆类营养品质 鉴定与评价). Beijing: China Agricultural Scientech Press, 1998(in Chinese)

[2] Long J-Y(龙静宜), Lin L-F(林黎奋), Hou X-S(侯修身). Food Legume Crops(食用豆类作物). Beijing: Science Press, 1989(in Chinese)

[3] Wang S-M(王述民), Tan F-J(谭富娟), Hu J-P(胡家蓬). Genetic diversity assessment of adzuki bean germplasm resources based on isozyme analysis. Sci Agric Sin(中国农业科 学), 2002, 35(11): 1311-1318(in Chinese with English abstract)

[4] Zheng Z-J(郑卓杰), Wang S-M(王述民), Zong X-X(宗绪晓). China Food Legume Science(中国食用豆类学). Beijing: China Agriculture Press, 1997. pp 173-195(in Chinese)

[5] Lin R-F(林汝法), Chai Y(柴岩), Liao Q(廖琴), Sun S-X(孙 世贤). Minor Grain Crops in China(中国小杂粮). Beijing: China Agricultural Scientech Press, 2002. pp 210-227(in Chinese)

[6] Van Hintum Th J L, Brown A H D, Spillane C, Hodgkin T. Core collections of plantgenetic resources. Rome: International Plant Genetic Resources Institute, 2000. pp 5-8

[7] Dalrymple D G. Development and spread of highyielding wheat varieties in developing countries, 7 th edn. Washington DC: US Agency for International Development, 1986

[8] Vellve R. Saving the Seed: Genetic Diversity and European Agriculture. London: Earthscan Publications, 1992

[9] Dowswell C R, Paliwal R L, Cantrell R L. Maize in the Third World. Boulder: Westview Press, 1996

[10] Jiang H-F(姜慧芳), Duan N-X(段乃雄). Utilization of groundnut germplasm resources in breeding programme. Crop Genet Resour(作物品种资源)，1998，(2)：24-25(in Chinese with English abstract)

[11] Shiv Kumar S, Gupta S, Chandra S, Singh B B. How wide is genetic base of pulse crops? In: Ali M, Singh B B, Shiv Kumar S, Dhar V, eds. Pulses in New Perspective. Proceedings of the National Symposium on Crop Diversification and Natural Resources Management, 20-22 December 2003. Kanpur: Indian Society of Pulses Research and Development, Indian Institute of Pulses Research, 2004. pp 211-221

[12] Upadhyaya H D, Furman B J, Dwivedi S L, Udupa S M, Gowda C L L, Baum M, Crouch J H, Buhariwalla H K, Singh S. Development of a composite collection for mining germplasm possessing allelic variation for beneficial traits in chickpea. Plant Genet Resour, 2006, 4: 13-19

[13] Bhattacharjee R, Khairwal I S, Bramel P J, Reddy K N. Establishment of a pearl millet [Pennisetum glaucum (L.) R. $\mathrm{Br}$.] core collection based on geographical distribution and quantitative traits. Euphytica, 2007, 155: 35-45

[14] Frankel O H. Genetic perspective of germplasm conservation. In: Arber W, Llimensee K, Peacock W J, Starlinger P, eds. Genetic Manipulations: Impact on Man and Society. Cambridge: Cambridge University Press, 1984. pp 161-170

[15] Holbrook C C, Anderson W F, Pittman R N. Selection of a core collection from the US germplasm collection of peanut. Crop Sci, 1993, 33: 859-861

[16] Prasada Rao K E, Ramanath Rao V. The use of characterization data in developing a core collection of sorghum. In: Hodgkin T, Brown A H D, van Hintum M J L, Morales E A V eds. Core Collections of Plant Genetic Resources. Chichester: A Wiley-Sayee Publications, 1995. pp 109-116

[17] Upadhyaya H D, Bramel P J, Singh S. Development of a chickpea core subset using geographical distribution and quantitative traits. Crop Sci, 2001, 41: 206-210

[18] Dong Y-C(董玉琛), Cao Y-S(曹永生), Zhang X-Y(张学勇), Liu S-C(刘三才), Wang L-F(王兰芬), You G-X(游光霞), Pang B-S(庞斌双), Li L-H(李立会), Jia J-Z(贾继增). Establishment of candidate core collections in Chinese common wheat germplasm. J Plant Genet Resour (植物遗传资源学报), 2003, 4(1): 1-8(in Chinese with English abstract)

[19] Li Y, Shi Y S, Cao Y S, Wang T Y. Establishment of a core collection for maize germplasm preserved in Chinese National Genebank using geographic distribution and characterization data. Genet Resour Crop Evol, 2004, 51: 845-852

[20] Reddy L J, Upadhyaya H D, Gowda C L L, Singh S. Development of core collection in pigeonpea [Cajanuscajan (L.) Millspaugh] using geographic and qualitative descriptors. Genet Resour Crop Evol, 2005, 52: 1049-1056

[21] Zhang X-J(张旭婧), Yu L-Q(于林清), Jia Z-B(贾志斌). Alfalfa core collection and establishment methology. Chin $J$ Grassland(中国草地学报), 2007, 29(1): 98-103(in Chinese 
with English abstract)

[22] Jia J-Z(贾继增), Zhang Q-F(张启发). Exploding gene resources for “the second green revolution”. China Basic Sci(中 国基础科学), 2001, 21(7): 4-8(in Chinese with English abstract)

[23] Li Z-C(李自超), Zhang H-L(张洪亮), Cao Y-S(曹永生), Qiu Z-E(表宗恩), Wei X-H(魏兴华), Tang S-X(汤圣祥), Yu P(余 萍), Wang X-K(王象坤). Studies on the sampling strategy for primary rice. Acta Agron Sin(作物学报), 2003, 29(1): 20-24(in Chinese with English abstract)

[24] Qiu L-J(邱丽娟), Cao Y-S(曹永生), Chang R-Z(常汝镇), Zhou X-A(周新安), Wang G-X(王国勋), Sun J-Y(孙建英), Xie H(谢华), Zhang B(张博), Li X-H(李向华), Xu Z-Y(许占 有), Liu L-H(刘立宏). Establishment of Chinese soybean $(G$. max) core collection: I. Sampling strategy. Sci Agric Sin(中国 农业科学), 2003, 36(12): 1442-1449(in Chinese with English abstract)

[25] Frankel O H, Brown A H D. Plant genetic resources today: A critical appraisal. In: Holden J H W, Williams J T eds. Crop Genetic Resources: Conservation and Evaluation. London: George Allen and Unwin, 1984. pp 249-257

[26] Brown A H D. Core collections: A practical approach to genetic resources management. Genome, 1989, 31: 818-824

[27] Wang S-M(王述民). The Core Collection Establishment and Its Genetic Diversity Assessment and Classification of Adzuki Bean [Vigna angularis (Willd) Ohwi \& Ohashi] Germplasm Resources. PhD Dissertation of China Agricultural University, 2001(in Chinese with English abstract)

[28] Cui Y-H(崔艳华), Qiu L-J(邱丽娟), Chang R-Z(常汝镇), Lü W-H(吕文河). Advances in research on core collection of plant germplasm resources. J Plant Genet Resour(植物遗 传资源学报), 2003, 4(3): 279-284(in Chinese with English abstract)

[29] Bisht I S, Mahajan R K, Patel D P. The use of characterization data to establish the Indian mungbean core collection and assessment of genetic diversity. Genet Resour Crop Evol, 1998, 45: $127-133$

[30] Maughan P J, Saghai M A, Buss G R. Microsatellite and amplified sequence length polymorphisms in cultivated and wild soybean. Genome, 1999, 38: 715-723

[31] Han L-D(韩立德), Xu H-M(徐海明), Hu J(胡晋). A study on representative evaluation parameters of quantitative traits for core collection. J Biomath (生物数学学报), 2006, 21(4): 603-609(in Chinese with English abstract)

[32] Ortiz R, Ruia-Tapia E N, Mijica-Sanchez A. Sampling strategy for a core collection of Peruvian quinoa germplasm. Theor Appl Genet, 1998, 96: 475-483

[33] Hu J-P(胡家蓬). Collection and evaluation of germplasm of adzuki bean (Vigna angularis) in China. Crop Genet Resour(作物品种资源), 1999, (1): 17-19(in Chinese with English abstract)

[34] Corley H C, William F A. Evaluation of a core collection to identify resistance to late leafspot in peanut. Crop Sci, 1995, 35: $1700-1720$

[35] Bayaa B, Erskine W, Singh M. Screening lentil for resistance to fusarium wilt: Methodology and sources of resistance. Euphytica, 1997, 98: 69-74

[36] Micklas P N, Delorme R, Hannan R, Dickson M H. Using a sub sample of the core collection to identify new sources of resistance to white mold in common bean. Crop Sci, 1999, 39: 569-573

[37] Upadhyaya H D, Ferguson M E, Bramel P J. Status of the Arachis germplasm collection at ICRISAT. Peanut Sci, 2001, 28: 89-96 